\title{
Factores Significativos en el Diseño de Proyectos Inmobiliarios de Tipo Industrial aplicando una Metodología General Ajustada
}

\author{
Oscar Palacio ${ }^{1}$, Rodrigo A. Britto ${ }^{2 \star}$ y Oscar Y. Buitrago ${ }^{1}$ \\ (1) Facultad de Ingeniería, Universidad Militar Nueva Granada, Programa de Ingeniería Industrial Carrera 11 No. 101 - \\ 80, Bogotá - Colombia (e-mail: oscar.palacio@unimilitar.edu.co) \\ (2) Facultad de Administración, Universidad de los Andes, Calle 21 No. 1-20, Bogotá - Colombia \\ (e-mail: ro-britt@uniandes.edu.co; oscar.buitrago@unimilitar.edu.co) \\ ${ }^{*}$ Autor a quien debe ser dirigida la correspondencia
}

Recibido Ago. 5, 2018; Aceptado Oct. 3, 2018; Versión final Dic. 15, 2018, Publicado Ago. 2019

\begin{abstract}
Resumen
El objetivo de este trabajo es establecer que factores son críticos para el diseño espacial de centros de distribución (DC), especialmente naves de almacenamiento inmóticas. Para esto, se utilizaron los principios de la metodología general ajustada (MGA) y el diseño de experimentos (DOE) en el contexto de una empresa de griferías en Bogotá, Colombia. Dicha metodología se basa en la aplicación de la MGA para proyectos inmobiliarios industriales, en un marco de redes de suministro. Adicionalmente, el diseño de experimentos (DOE) se empleó como forma de obtener la alternativa óptima que satisfaga las expectativas de la empresa y sus clientes desde el punto de vista económico y estadístico. En los resultados se encontró que la capacidad de almacenamiento del centro de distribución centralizado (CDC) es el factor más significativo en la etapa de diseño, con lo anterior se concluye que, esta característica de un CDC es esencial para racionalizar la inversión en capital de trabajo y maximizar los beneficios de la empresa.
\end{abstract}

Palabras clave: logística; metodología general ajustada; proyecto inmobiliario industrial; red de suministro;

\section{Significant Factors in the Design of Industrial Real Estate Projects applying an Adjusted General Methodology}

\begin{abstract}
The objective of this paper is to establish what are the critical factors for the spatial design of distribution centers, specially inmotic storage ships. To do so, the principles of the adjusted general methodology (MGA) were used along with the implementation of experimental design (DOE) in the context of a faucet production company in Bogotá, Colombia. The MGA methodology lays its foundations in the application to industrial real estate projects, in a supply network scheme. Furthermore, DOE is used as a way of obtaining an optimal alternative which satisfies the expectations of the company and its clients from an economic and statistical point of view. The results show that the storage capacity of the centralized distribution center is the most significant factor in the design stage, being an essential factor for rationalizing investment in working capital and for maximizing the benefits of the company.
\end{abstract}

Keywords: logistics; adjusted general methodology; industrial real estate projects; supply networks 


\section{INTRODUCCIÓN}

Palacio-León (2012), sostiene que "Integrar, sincronizar y coordinar las funciones fundamentales de la firma, con el objetivo de brindar un nivel de servicio adecuado, a unos costos razonables, tiene una importancia estratégica al interior de la Red de Suministro Dirigida por la Demanda (DDSN, por sus siglas en inglés)". Más aún, los agentes que integran la DDSN comparten una mayor cantidad de datos para poder responder de manera más eficiente a todos los requerimientos del mercado y utilizan la Técnica Pull (Gnimpieba, 2015). La importancia estratégica de las funciones antes mencionadas ha permitido que tanto compradores como vendedores pertenecientes a una misma DDSN, implementen en sus procesos de negociación cotidianos metodologías basadas en aspectos no solo colaborativos sino también cooperativos en gestión de almacenes, a partir de una política de suministro consensuada que genere valor compartido entre los interesados (PalacioLeón, 2019). Aspectos que hacen parte de las consideraciones en una Metodología General Ajustada (MGA) para Proyectos Inmobiliarios de Tipo Industrial (PITI), utilizada en el diseño estratégico de centros de distribución (CD) para productos no perecederos, a partir de datos de inventario conjunto, que es la que se propone y se presenta en este trabajo. La Metodología General Ajustada (MGA) es la herramienta gerencial utilizada para evaluar de forma eficiente y rápida los proyectos de inversión pública en Colombia en donde las características específicas, tales como las financieras, técnicas, ambientales y sociales de éste, son revisadas y aprobadas, de acuerdo los lineamientos establecido en el MGA (Mejía y Franco-Duran, 2017).

La MGA para PITI con enfoque en CD, está orientada hacia la configuración física de una Red de Suministros (RdS) que atiende la distribución de productos no perecederos. Habría que decir también, se compone de un modelo de negocio equilibrado, apoyado en una arquitectura empresarial coherente, con la planeación estratégica conjunta que implementen los agentes pertenecientes a la misma, con el objeto de detectar y reaccionar ante las señales de la demanda en tiempo real y que permita cumplir con las expectativas del mercado objetivo en cuanto a nivel de servicio, en total respecto por la cultura organización y gobernanza que adapta cada uno de los agentes que integran (Palacio-León, 2019; Palacio-León, 2012). Por otra parte, la DDSN se caracteriza por el desarrollo de los siguientes procesos operativos (Mendes, 2011): i) administración de la demanda; ii) cumplimiento del servicio; y iii) gestión de pagos. Accionada por los clientes finales, la demanda se articula a través de la colocación de los pedidos que se propagan por el canal de ventas directo y/o por intermediación. El cumplimiento en las entregas de las órdenes de pedido obedece al balance de la unidad de carga eficiente (UCE), coordinada entre los procesos físicos de manufactura, almacenamiento y transporte aguas arriba de la RdS. La gestión de pagos culmina con las transacciones que transfieren los fondos hacia los proveedores de los bienes y servicios.

Todavía cabe señalar que Palacio-León (2012), considera que son objetivos de la gestión de almacenes "la satisfacción del cliente, en cuanto al cumplimiento de los requerimientos del nivel de servicio al cliente, la efectividad de los costos de operatividad y la manutención de los materiales administrados por el sistema de almacenamiento (WMS, por sus siglas en inglés)". La función de un WMS es la de garantizar la custodia del material (inventario) por un período de tiempo razonable y permitir acceder a él cuándo sea requerido (Mauleón, 2013; Baker y Canessa, 2009). El WMS proporciona herramientas e información necesaria para gestionar, en forma adecuada, el espacio disponible del almacén procurando maximizar la satisfacción del cliente, el espacio de trabajo y el sistema de almacenaje del inventario (Bartholdi III y Hackman, 2017; Tompkins, 2010). (Vélez-Pareja, 2013; Charvat, 2003). Además, los PITI están definidos por una serie de actividades concurrentes que requiere un esfuerzo temporal de trabajo, que se lleva a cabo en forma única e irrepetible, para crear un producto estratégico de conocimiento de calidad superior con características complejas (Palacio-León, 2019). En el proyecto se evalúa en función de su ciclo de inversión y financiamiento, en atención a las siguientes características: i) tiene una fecha de inicio y determinación conocida; ii) posee restricciones en programación, costos y calidad; iii) está expuesto al riesgo; y iv) tiene un alcance definido (Vélez-Pareja, 2013; Charvat, 2003). El objetivo que persigue un PITI es satisfacer la necesidad de edificación de instalaciones industriales (oficinas, plantas de producción, naves de almacenamiento, entre otros) que sean amigables con el medio ambiente, y que cumplan con los principios inmóticos de constructibilidad (Palacio-León, 2012; Sule, 2008).

Conviene subrayar, que un PITI debe garantizar los siguientes principios durante todo su ciclo de vida: i) principio de la integración de conjunto; ii) Principio de la mínima distancia recorrida; iii) principio de la circulación o flujo de materiales; iv) principio en igualdad de condiciones; v) principio del espacio cúbico; y vi) principio de la flexibilidad (Diego-Más, 2006). Además, deberá cumplir con los estándares de cargas energéticas y sustentabilidad ambiental regulados por la ISO 14040 y sus normas reglamentarias de apoyo relacionadas con el periodo de posesión y operación de instalaciones industriales, el cual deberá superar los 50 años como mínimo (Sule, 2008). Algunos trabajos de la revisión de la literatura en diseño de bodega han encontrado importantes aspectos relacionados con la metodología utilizada y otros señalan la importancia del tema en Colombia. Por ejemplo, Baker y Canessa (2009) exploran las diferentes metodologías utilizadas para diseñar almacenes. Estos autores, enfatizan la importancia de los almacenes y su diseño dentro de una 
cadena de suministro y señalan el papel vital que desempeñan en el éxito de las empresas modernas. Los autores indican que no hay una manera perfecta para diseñar almacenes y que hay poca literatura sobre este tema. Restrepo y Uribe (2002) identifican los criterios económicos más relevantes con el fin de determinar la viabilidad económica de un proyecto de plantas industriales. Por su parte, Hualpa y Suárez (2013) afirman que existen pocos estudios especializados en este tema en Colombia, principalmente porque la literatura colombiana en este tema se enfoca en la gestión de la cadena de suministro. Por lo tanto, consideran que es un tema importante para futuras investigaciones en el país. En este artículo se determinan los factores críticos en el diseño espacial de DC, partiendo de un diseño metodológico en el que se describen los supuestos, variables y parámetros determinados en una investigación adelantada durante el último quinquenio con expertos en almacenamiento de la ciudad de Bogotá (Palacio-León, 2012). Con la metodología propuesta se obtienen diferentes alternativas de diseño para un $C D$ totalmente nuevo, requerido por una empresa del sector de grifería en Colombia, que son evaluadas en términos del beneficio actualizado neto ajustado (BANA), a una tasa de interés de oportunidad (TIO) del $5 \%$ efectiva anual.

Para determinar los factores considerados que son significativos en el diseño del CD, para así centrarse en su optimización y poder obtener la alternativa que desde el punto de vista económico y estadístico satisfaga de mejor manera las expectativas de cooperación entre los dos eslabones consecutivos de la RS considerada, se utiliza el diseño de experimentos (DOE, por sus siglas en inglés). El diseño de experimentos en esta metodología tiene como ventajas el identificar interacciones significativas entre factores de diseño (que también se consideran en la etapa de optimización) y que los niveles (valores) que tomen los factores no significativos se pueden establecer a conveniencia del diseñador. También permite obtener deducciones válidas y relevantes en forma eficiente y económica (Montgomery, 2013), aun considerando la variabilidad presentada, al repetir un experimento en condiciones idénticas, (Kuehl, 1999) pues incrementa la probabilidad de detección de cambios significativos que experimenta la variable de respuesta; de esta forma el investigador incrementa su conocimiento frente al comportamiento del proceso de su interés (Kolaczyk, 2009; Gatignon, 2014). Es importante resaltar que el diseño de experimentos es un enfoque válido ya que ha sido utilizado en varios estudios para el diseño de bodegas (Altarazi y Ammour, 2017; Chackelson, 2013; Ekren, 2010). En cuanto a la estructura de este artículo, se inicia con una descripción de la metodología empleada con soporte en diseño de experimentos, a fin de establecer que factores son críticos para el diseño espacial de CD, en especial para naves de almacenamiento inmóticas. Posteriormente se presentan y discuten los resultados para culminar con las conclusiones.

\section{METODOLOGÍA}

Los pasos metodológicos se definen teniendo en cuenta: a) el uso de la MGA para la formulación de un PITI destinado al diseño de un CD totalmente nuevo; b) una vida útil que supere por lo menos cincuenta años; c) la MGA considera los efectos que tiene la localización del muelle de carga y descarga de material, el diseño adoptado por las bahías de almacenamiento, el nivel de inventario máximo proyectado y la estrategia de almacenamiento, valorados en términos del costo de operación y posesión proyectado para el CD. El nivel del inventario almacenado en el multi-eslabón comprador-vendedor es conocido y el tiempo de respuesta es pactado al inicio de la negociación, la administración del pedido es conjunta siguiendo las estrategias puras de coordinación de inventarios o una combinación de ellas, ya sea para mono-productos o multi-productos (Jiménez, 2005; Tersine, 1994).

Para la evaluación y comparación del desempeño sostenible, de cada una de las propuestas de diseño del PITI, se empleó como criterio económico de decisión el BANA contra la tasa verdadera de rentabilidad (TVR), en un horizonte de diez años, (Vélez-Pareja, 2013), teniendo en cuenta los siguientes cuatro factores:

1) el PITI fue localizado con antelación, estudio que cobijó una evaluación dinámica (modelado matemático a nivel macro, meso y micro localización) afianzada con una evaluación estática (Plan de Ordenamiento Territorial);

2) La altura de la nave de almacenamiento está entre ocho y doce metros a partir de caída de aguas, construida modularmente con principios de inmótica, planimetría de planta y con una cubierta a dos aguas. Este parámetro es el que define las dimensiones de la nave de almacenamiento y las inversiones mostradas en la Fig.1;

3) El área del edificio define el área del terreno proyectado (área de bodega más patio de maniobras); y

4) En general, la planificación estratégica del sistema productivo se sustenta en las siguientes reglas de diseño de edificios industriales (Palacio-León, 2019): i) Regla de Pre-Visión del Uso del Diseño Espacial del 85/100; y ii) la ausencia de reglas no solo universales sino también prácticas de diseño espacial de instalaciones industriales. 


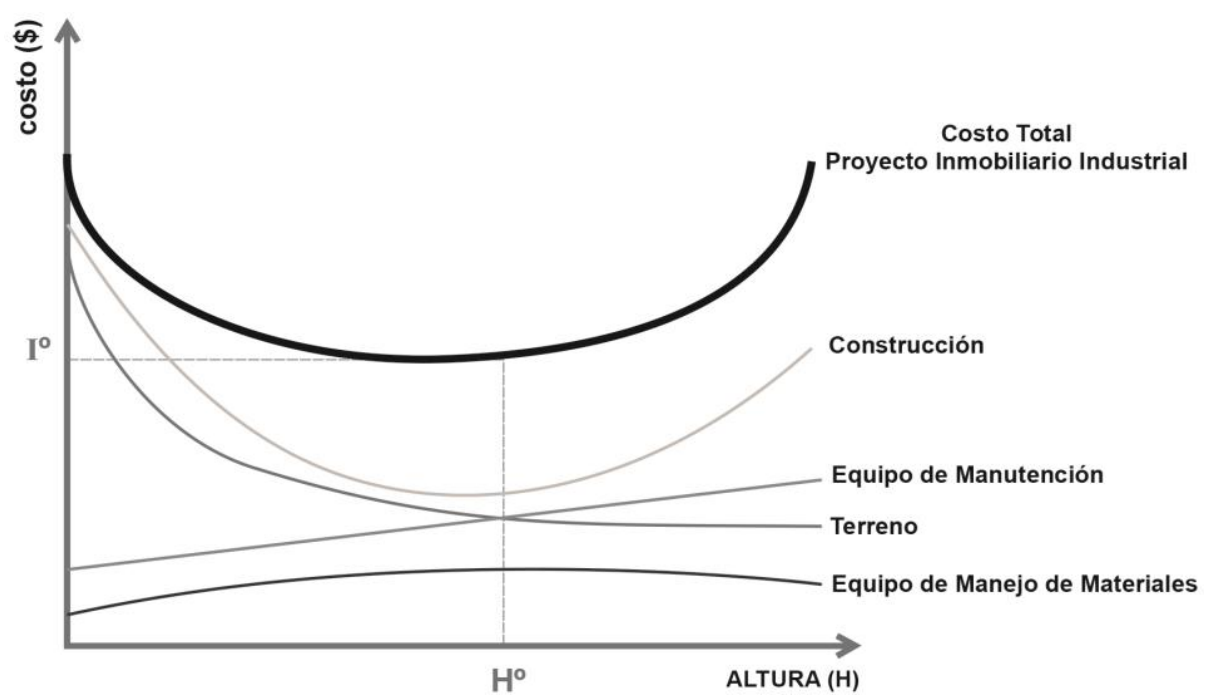

Fig. 1: Relación entre Inversión y Altura del CD (Tomada de Palacio-León, 2012)

\section{Marco del modelo metodológico}

La metodología para el emplazamiento inmótico de naves de almacenamiento sostenible ajustada (MEINASA) empleada, comprende los siguientes perfiles: i) diseño de una nave de almacenamiento (NdA) nueva; ii) expansión o traslado a una $\mathrm{NdA}$; iii) reordenación del layout de una $\mathrm{NdA}$; y iv) ajustes menores al ordenamiento espacial en NdA, (Palacio-León, 2012; Diego-Más, 2006). MEINASA es una MGA orientada al proceso que se adapta con facilidad a técnicas de chequeo diagnósticas como SCAMPER (sustituir, combinar, adaptar, modificar, poner en otros usos, eliminar, reorganizar) que es una técnica de creatividad desarrollada finalizando la década de los cincuenta para procesos de mejora continua. De esta fusión, surge el modelo metodológico general ajustado para el diseño de sistemas productivos destinados al bodegaje de productos no perecederos que se describe a continuación.

\section{Cuantificación del inventario máximo conjunto proyectado}

Se parte del supuesto que el inventario máximo actual se cuantifica independientemente por parte de cada agente logístico a partir de las condiciones de negociación pactadas, que contempla como mínimo los siguientes factores de modelado: i) los consumos del comprador siguen un patrón estocástico y monitoreados con sistemas EVTC sincrónicos por parte del vendedor; ii) se emplea la misma estrategia de almacenamiento; iii) la proyección del cubo de almacenamiento sigue el mismo comportamiento para la dupla vendedorcomprador (índice de volumen interior de almacenamiento); iv) se emplea la misma técnica de clasificación ABC más $D$ del inventario en unidades monetarias por familia de productos; iv) se establece el mismo nivel de servicio deseado por familia de productos; v) se estipula la misma unidad básica de almacenamiento para el sistema vendedor-comprador; vi) se costea el sistema de almacenamiento por parte de cada agente logístico en forma independiente; y vi) se establece la política de coordinación del inventario que minimice el costo total conjunto de la operación como tal.

\section{Cubicaje del SKU de almacenamiento}

Este proceso consiste en dimensionar la unidad básica de almacenamiento (SKU) en función de: i) las características del producto a almacenar; ii) flujo de materiales en $\mathrm{t} / \mathrm{m}^{3} / \mathrm{SKU}$; ii) el uso del cubo; iii) la política de coordinación del inventario negociada entre vendedor-comprador; iv) el cumplimiento de la normativa vigente, entre otros aspectos (Palacio-León, 2019). En la situación estudiada se estandarizó el elemento de manutención del inventario (pallet universal), teniendo en cuanta las dimensiones y características de los elementos a almacenar y se definió estibarlos con una UCE homogenizada volumétricamente con un peso comprendido entre los 200 a 500 kilogramos.

\section{Área de bodegaje}

Teniendo en cuenta la inversión requerida en terrenos, construcción del edificio industrial y equipo de manutención y almacenamiento del inventario (Lahmar, 2008), se valida la altura a partir de caída de aguas, que es considerado el parámetro fundamental de diseño del CD. Este se contrasta con la operatividad del WMS, diseñado para el CD en términos de: i) localización del muelle de carga y descarga de mercancías; ii) orientación de las bahías de almacenamiento de acuerdo con el modelo de Bassan; iii) margen de error en el 
diseño; y iv) proyección del edificio a partir de índices de bodegaje (Palacio-León, 2019; Rakesh y Adil, 2015; Mauleón, 2013; Palacio-León, 2012). Siguiendo los criterios de Mauleón (2013) para el dimensionamiento de los rack con pallet universal, se establece que en la situación analizada, para 12 pallets por módulo de almacenamiento, las dimensiones de la bahía de almacenamiento doble son ancho de 3,8 m; profundidad de $5,9 \mathrm{~m}$ y altura de $1,85 \mathrm{~m}$.

\section{Terreno proyectado para el CD}

El terreno proyectado consta del cálculo del área de los muelles para carga y descarga de mercancías y el patio de maniobras, considerando el medio de transporte seleccionado para la operación de distribución, más el área calculada para el CD, considerando aspectos (Palacio-León, 2012; Lahmar, 2008): i) legales (POT municipal); ii) técnicos; y iii) ambientales. Una vez definidos los parámetros de diseño a emplear y conociendo el área del terreno disponible, se puede calcular el área del edificio utilizando las relaciones de Muther (1968), mostradas en la Tabla 1.

Tabla 1: Relación del área del Terreno/Edificio Industrial (Datos tomados de Muther, 1968)

\begin{tabular}{|c|c|c|c|c|c|c|c|c|c|c|c|}
\hline $\begin{array}{c}\text { Dimensiones de los } \\
\text { edificios }\end{array}$ & \multicolumn{9}{|c|}{ Superficies necesarias en función de la relación [ratio] (terreno-edificio) elegida } \\
\hline$m^{2}$ & ha & $1 / 1$ & $2 / 1$ & $3 / 1$ & $4 / 1$ & $5 / 1$ & $6 / 1$ & $7 / 1$ & $8 / 1$ & $9 / 1$ & $10 / 1$ \\
\hline 500 & & 1000 & 1500 & 2000 & 2500 & 3000 & 3500 & 4000 & 4500 & 5000 & 5500 \\
\hline 1000 & & 2000 & 3000 & 4000 & 5000 & 6000 & 7000 & 8000 & 9000 & 10000 & 11000 \\
\hline 2000 & & 4000 & 6000 & 8000 & 10000 & 12000 & 14000 & 16000 & 18000 & 20000 & 22000 \\
\hline 3000 & & 6000 & 9000 & 12000 & 15000 & 18000 & 21000 & 24000 & 27000 & 30000 & 33000 \\
\hline 4000 & & 8000 & 12000 & 16000 & 20000 & 24000 & 28000 & 32000 & 36000 & 40000 & 44000 \\
\hline & & & & & & & & & & & \\
\hline 5000 & 0.5 & 1 & 1.5 & 2 & 2.5 & 3 & 3.5 & 4 & 4.5 & 5 & 5.5 \\
\hline 6000 & 0.6 & 1.2 & 1.8 & 2.4 & 3.0 & 3.6 & 4.2 & 4.8 & 5.4 & 6 & 6.6 \\
\hline 7000 & 0.7 & 1.4 & 2.1 & 2.8 & 3.5 & 4.2 & 4.9 & 5.6 & 6.3 & 7 & 7.7 \\
\hline 8000 & 0.8 & 1.6 & 2.4 & 3.2 & 4.0 & 4.8 & 5.6 & 6.4 & 7.2 & 8 & 8.8 \\
\hline 9000 & 0.9 & 1.8 & 2.7 & 3.6 & 4.5 & 5.4 & 6.3 & 7.2 & 8.1 & 9 & 9.9 \\
\hline & & & & & & & & & & & \\
\hline 10000 & 1 & 2 & 3 & 4 & 5 & 6 & 7 & 8 & 9 & 10 & 11 \\
\hline 20000 & 2 & 4 & 6 & 8 & 10 & 12 & 14 & 16 & 18 & 20 & 22 \\
\hline 30000 & 3 & 6 & 9 & 12 & 15 & 18 & 21 & 24 & 27 & 30 & 33 \\
\hline 40000 & 4 & 8 & 12 & 16 & 20 & 24 & 28 & 32 & 36 & 40 & 44 \\
\hline 50000 & 5 & 10 & 15 & 20 & 25 & 30 & 35 & 40 & 45 & 50 & 55 \\
\hline 100000 & 10 & 20 & 30 & 40 & 50 & 60 & 70 & 80 & 90 & 100 & 110 \\
\hline
\end{tabular}

\section{Evaluación financiera de alternativas de inversión}

Se evalúan las alternativas de inversión suponiendo: i) proyectos mutuamente excluyentes; ii) vidas iguales; iii) valor de mercado de las inversiones no capitalizadas; iv) tasa de oportunidad calculada acorde al valor de colocación de dinero del sector financiero colombiano; v) evaluación del flujo de caja en pesos constantes; vi) ingreso neto operacional unitario del servicio calculado con el margen sobre el precio de venta (PalacioLeón, 2019; Vélez, 2006).

\section{Caracterización del centro de distribución y manejo de inventarios}

El diseño de la red de centros de distribución al interior de una RS orientada a la distribución de productos no perecederos, se puede caracterizar principalmente por la existencia de un CD centralizado (CDC) y varios puntos de venta filiales (PVF). El CDC es el puente de conexión entre la cadena interna y externa de suministros de productos no perecederos de una RS específica del mundo real. En otras palabras, este CDC es el encargado de alimentar las existencias de productos no perecederos requeridos dentro de la RS, mientras que cada uno de los PVF lo hace para un nivel de servicio particularizado. Específicamente en este trabajo se proponen diseños para CDC, a partir de datos de inventario conjunto, donde el multi-eslabón vendedor-comprador establecen, de común acuerdo, una política de coordinación eficiente basada en la 
minimización del costo promedio de ordenar y conservar inventario en el CDC y en cada uno de los PVF. Dentro de las preguntas que se buscan responder al diseñar un $C D$ en general con datos de inventarios coordinado, se encuentra la determinación del tamaño de lote óptimo y los tiempos de reabastecimiento que permitan el uso racional del cubo de almacenamiento para cada uno de los niveles de la RS intervenida.

\section{Coordinación de inventarios}

Es un proceso de negociación bilateral multi-paramétrica de compraventa de productos multi-eslabón clienteproveedor (Albrecht, 2010; Jiménez, 2005). Es decir, el agente comprador define sus requerimientos de compra y preferencias mediante restricciones, y el agente vendedor tiene definida la utilidad que le reporta la venta de cada uno de los productos que hacen parte de su portafolio de servicio. Existen modelos determinísticos y estocásticos incorporables en los procesos de negociación bilateral multi-paramétricos (Albrecht, 2010; Tersine, 1994), pero la obtención de políticas óptimas de reposición de inventarios, en sistemas multi-eslabón, sigue siendo un problema complejo, Jimenez (2005). También se han desarrollado técnicas de solución cuyo propósito es determinar la cantidad óptima de pedido para el caso multi-producto, un CDC y (n) PVF (Abdul-Jalbar, 2006; Muckstadt y Roundy, 1987). Dado que el centro de interés de este trabajo es establecer que factores son críticos durante el diseño espacial de un CD de características inmóticas, se desarrolló una simulación de un sistema de suministro conformado por un CD que soporta los requerimientos de cinco administradores de cuentas claves filiales (ACCF), con respecto a un SKU homogéneo, el cual, sigue una estrategia de coordinación para la gestión de inventarios justo a tiempo (JAT) (Jimenez, 2005), buscando una política conjunta de inventarios que minimice el costo promedio de mantenimiento y solicitud de órdenes de pedido entre el CDC y los ACCF considerados.

Los supuestos base, sobre los que se desarrolla esta estrategia son (Palacio-León, 2012): i) tasas de consumo para los ACCF estocásticas; ii) el actor logístico que tiene el poder en la RS es el AC; iii) reposición de productos instantánea; iv) costos de conservación de inventario y un cargo fijo por orden colocada en cada entidad; v) no se permiten pedidos atrasados, ventas pérdidas ni faltantes; vi) el consumo se cuantifica en base diez con una aproximación a partir del $30 \%$; vii) el SKU se administra para cada ACCF con el esquema JIT de los dos contenedores; y viii) los tiempos de imprevistos se asumen despreciables. Se seleccionó el enfoque de coordinación del modelo de intervalo económico de pedido para múltiples ítems, extendido a un sistema justo a tiempo, EOIESJIT (Palacio-León y Adarme-Jaimes, 2014), que, en general, consiste en determinar una longitud de ciclo común para el almacenamiento coordinado para diferentes SKU homogéneos administrados por un solo agente logístico (Palacio-León, 2012). Este modelo de coordinación de inventarios permite determinar un tiempo único de reabastecimiento para el CDC, considerando los tiempos de ciclo de cada ACCF como múltiplos enteros del mismo. Esta aproximación se caracteriza por permitir que cada ítem o, en este caso, cada ACCF puedan administrar una longitud de ciclo diferente. Esto soportado en sistemas EVTC sincrónicos, demanda estocástica, tiempos de reabastecimiento comunes y se pactan envíos de SKU homogéneos en un lapso común, tal y como ocurre en sistemas de Justo a Tiempo (JAT).

\section{SIMULACIÓN Y EVALUACIÓN ESTADÍSTICA}

Se emplea el diseño experimental para determinar la significancia de los factores principales y sus interacciones en la variable de respuesta (BANA) con que se evalúan las propuestas de diseño del CDC. Se utiliza un diseño factorial $2^{k}$ replicado ya que son considerados como una forma eficiente de realizar la experimentación (Czitrom y Spagon, 1997) y también este tipo de diseño $\left(2^{5}\right)$ se aplicó en bodegas de minoristas, empleando un esquema de pasos de eliminación sucesiva para obtener la ecuación respectiva (Chackelson, 2013). En la Tabla 2 se muestran los factores del diseño $2^{4}$ junto con su notación, a partir del cual se simularon los correspondientes flujos de caja que permitieron obtener como variable de respuesta el BANA teniendo en cuenta los ingresos y egresos asociados a cada combinación de factores (experimento). Con los resultados de la simulación, se recurrió al software SPSS ${ }^{\circledR}$ Statistics versión 21, para mediante la aplicación de pasos de eliminación sucesiva llegar a una ecuación que incluya únicamente los factores más significativos en el modelo.

Tabla 2: Factores estudiados

\begin{tabular}{|l|c|c|}
\hline \multirow{2}{*}{ Factor } & \multicolumn{2}{c|}{ Niveles } \\
\cline { 2 - 3 } & Alto & Bajo \\
\hline Tamaño Proyectado del CDC (A) & Máximo & Mínimo \\
\hline Criterio de Costeo del Servicio (B) & Posición & Pallet \\
\hline Política de Almacenamiento (C) & Dedicado & Caótico \\
\hline Capacidad de Bodegaje (D) & Máxima & Mínima \\
\hline
\end{tabular}




\section{Caso de aplicación}

La metodología propuesta se aplicó al caso de una compañía líder del sector de grifería de Bogotá D.C que está interesada en la construcción de un CDC para satisfacer un mercado anual de 50.000.000 USD (modelado para la simulación como una variable aleatoria distribuida normalmente, $N(\mu=50.000 .000$ USD/año, $\sigma=3.000 .000 \mathrm{USD} /$ año), la cual se estima que crezca en promedio $2 \%$ anual durante un horizonte de diez años. Por su parte, la junta directiva de la compañía reservó una partida de financiamiento conformada exclusivamente por recursos propios para este PITI. El capital será invertido en la compra de un terreno de 20 hectáreas (que está disponible para la venta en el sitio indicado en el estudio de microlocalización) y en la edificación de una NA con un aforo para al menos 30.000 pallets universales.

\section{Definición de los parámetros de diseño del CDC}

EI CDC se diseña con una altura de 10 metros a partir de caída de aguas. Las inversiones que demanda la construcción fueron estimadas, así: i) costo promedio del encerramiento $1.000 \mathrm{USD} / \mathrm{m}^{2}$; ii) costo de la cubierta $110 \mathrm{USD} / \mathrm{m}^{2}$; iii) costo del terreno $550 \mathrm{USD} / \mathrm{m}^{2}$; iv) costo del sistema inmótico $170 \mathrm{USD} / \mathrm{m}^{2} ;$ y v) margen de error empleado en la proyección del dimensionamiento de la NA 10\%. Los pallets se estiban con una UCE homogenizada volumétricamente con un peso comprendido entre los 200 a 500 kilogramos. Este cubicaje representa un ingreso neto operacional promedio de 250 USD por pallet. Los costos administrativos se distribuyen por pallet, así: i) preparación de pedidos 50 USD/Año; ii) tarifa de alistamiento por ACCF 5 USD/Año-ACCF; iii) tasa de transferencia del $30 \%$ anual; y iv) el inventario de seguridad equivale a un $15 \%$ del nivel de la ocupación del área de bodegaje.

Por su parte, el dimensionamiento de la bahía de almacenamiento doble se proyecta con un ancho de 3,8 $\mathrm{m}$ una profundidad de $5,9 \mathrm{~m}$, altura de $1,85 \mathrm{~m}$ y 12 pallets por nivel. El costo promedio estimado por nivel doble de rack es de 560 USD. El sistema de mantenimiento y manejo de materiales requiere la implementación de un paquete informático (WMS) a un costo de 175.000 USD/Licencia. Se necesitan tres carretillas retráctiles a un costo de 75.000 USD/Unidad. El costo promedio de un pallet universal es de 25 USD. Se estima un costo adicional promedio de manejo de materiales de 1,6 USD/m-pallet. Por política de la firma el SKU de bodegaje es el mismo que la UCE de distribución, motivo por el cual no requiere preparación de pedidos, por lo que la totalidad de los huecos disponibles serán para almacenar pallets completos.

El sistema de almacenamiento de la compañía se evalúa económicamente a 10 años, periodo comúnmente utilizado, Palacio-León (2012), en lo referente a suelos, edificio, sistema inmótico y racks. Mientras que para las carretillas y el sistema WMS la evaluación es a cinco años, (Mauleon, 2013). La RS está conformada por cinco ACCF, sobre los cuales recae la administración de la demanda, el cumplimiento del servicio y la gestión de pagos del negocio. Los requerimientos de inventario de consumo por parte de los ACCF se muestran en la Tabla 3. El intervalo económico de pedido conjunto es de una semana y el inventario de consumo oscila entre 9.000 y 10.240 pallets dependiendo de la política de almacenamiento pactada entre los ACCF y el CDC que conforman la RS. En términos generales, los ACCF demandan espacio para bodegaje de 22.294 pallets, en el caso de involucrar en el proceso de coordinación una política de almacenamiento dedicada durante el primer año de operación, mientras que, para el caso contrario, solo serían requeridos 21.047 pallets.

Tabla 3: Inventario máximo demandado por los ACCF

\begin{tabular}{|l|c|c|c|c|}
\hline \multirow{2}{*}{ ACCF } & \multirow{2}{*}{$\begin{array}{c}\text { Lead time } \\
\text { (semanas) }\end{array}$} & \multirow{2}{*}{$\begin{array}{c}\text { Participación en la } \\
\text { demanda anual (\%) }\end{array}$} & \multicolumn{2}{c|}{\begin{tabular}{c} 
Política de almacenamiento (Pallets) \\
\cline { 4 - 5 }
\end{tabular}} \\
\cline { 4 - 5 } & 2 & 25 & 4079 & Caótica \\
\hline Local & 2 & 5 & 817 & 3767 \\
\hline Regional centro & 4 & 25 & 6795 & 6483 \\
\hline Nacional zona 1 & 4 & 30 & 8156 & 7782 \\
\hline Nacional zona 2 & 2 & 15 & 2447 & 2260 \\
\hline Institucional & & & &
\end{tabular}

Este modelo de negocio le cuesta a la firma en promedio el $35 \%$ anual de los ingresos operacionales. Los costos de operación y posesión del CDC corresponden al $40 \%$ de los ingresos. Las subvenciones se dan por cinco años por ser un proyecto productivo totalmente nuevo y corresponde al $25 \%$ de los impuestos en ese mismo periodo de tiempo pagaderos anualmente. El capital de trabajo está representado por la nómina de colaboradores y por el costo fijo de compra de los pallets del CDC. Las variaciones hacen referencia al valor de mercado neto de los activos al final del año diez. Los aspectos estructurales, de infraestructura y locativos están fuera del alcance de este diseño metodológico. 


\section{RESULTADOS Y DISCUSIÓN}

En la Tabla 4 se reportan los valores promedio obtenidos para el BANA en cada tratamiento (simulaciones ejecutadas aleatoriamente en hoja de cálculo, con macros codificados en Visual Basic Aplication para Excel $\left.{ }^{\circledR}\right)$, después de haber aplicado la MGA para el PITI al correspondiente diseño del CDC en el caso considerado y con un diseño de experimentos $2^{\mathrm{k}}$ (ya aplicado a bodegas de minoristas por Chackelson, 2013).

Tabla 4: Promedio de la variable de respuesta para cada tratamiento

\begin{tabular}{|c|c|c|c|c|c|}
\hline \multirow{3}{*}{$\begin{array}{l}\text { Proyección del } \\
\text { tamaño del } \\
C D C\end{array}$} & \multirow{3}{*}{$\begin{array}{c}\text { Criterio de } \\
\text { costeo del } \\
\text { servicio }\end{array}$} & \multicolumn{4}{|c|}{ Política de almacenamiento } \\
\hline & & \multicolumn{2}{|c|}{ Dedicado } & \multicolumn{2}{|c|}{ Caótico } \\
\hline & & $\begin{array}{l}\text { Capacidad } \\
\text { mínima de } \\
\text { bodegaje }\end{array}$ & $\begin{array}{c}\text { Capacidad } \\
\text { máxima de } \\
\text { bodegaje }\end{array}$ & $\begin{array}{l}\text { Capacidad } \\
\text { mínima de } \\
\text { bodegaje }\end{array}$ & $\begin{array}{c}\text { Capacidad máxima } \\
\text { de bodegaje }\end{array}$ \\
\hline \multirow{2}{*}{ Mínima } & Posición & USD $66,821,550$ & USD $31,980,930$ & USD $61,087,110$ & USD $43,027,370$ \\
\hline & Pallet & USD $65,369,870$ & USD $29,893,700$ & USD $59,524,340$ & USD $41,087,170$ \\
\hline \multirow{2}{*}{ Máxima } & Posición & USD $36,615,900$ & USD $55,602,040$ & USD $21,092,780$ & USD $42,731,700$ \\
\hline & Pallet & USD $34,215,930$ & USD $37,227,290$ & USD $16,783,090$ & USD 41,087,170 \\
\hline
\end{tabular}

Todos los resultados presentados en la Tabla 4 son económicamente factibles, siendo la mejor alternativa la asociada la proyección del tamaño del CDC mínimo, con política de almacenamiento dedicada, capacidad mínima de bodegaje y con un sistema de facturación del servicio en función de la posición. Los resultados del correspondiente análisis de varianza se presentan en la Tabla 5 con un nivel de significancia del $5 \%$.

Tabla 5: Resultados del análisis de varianza

\begin{tabular}{|c|c|c|}
\hline Efecto & Coeficiente & $\mathrm{t}$ \\
\hline $\mathrm{A}$ & -2.23 & -1.88 \\
\hline $\mathrm{B}$ & -1.98 & -1.67 \\
\hline $\mathrm{C}$ & -2.22 & -1.87 \\
\hline $\mathrm{D}$ & -6.97 & $-5.91^{\star}$ \\
\hline $\mathrm{AB}$ & 3.60 & $3.05^{\star}$ \\
\hline $\mathrm{AC}$ & -0.90 & -0.76 \\
\hline $\mathrm{AD}$ & 10.85 & $9.20^{\star}$ \\
\hline $\mathrm{BC}$ & 0.85 & 0.72 \\
\hline $\mathrm{BD}$ & -3.40 & $-2.88^{*}$ \\
\hline $\mathrm{CD}$ & -1.40 & -1.18 \\
\hline Constante & 42.60 & $37.11^{*}$ \\
\hline
\end{tabular}

De acuerdo con la Tabla 5, el único efecto simple que es significativo es la capacidad de bodegaje (D), también lo son tres interacciones dobles: La del tamaño proyectado del CDC con el criterio de costeo del servicio (AB), la del tamaño proyectado del CDC con la capacidad de bodegaje (AD) y la del criterio de costeo del servicio con la capacidad de bodegaje (CD). La ecuación (1) corresponde al modelo extenso e incluye todos los efectos analizados.

$$
B A N A=42.60-2.23 A-1.98 B-2.22 C-6.97 D+3.60 A B-0.90 A C+10.85 A D+0.85 B C-3.40 B D-1.40 C D
$$

Finalmente, a partir del modelo extenso (1), se emplea un esquema de pasos de eliminación sucesiva (en el que se descartan términos de acuerdo con la contribución a la significancia del modelo) hasta obtener la ecuación (2), que permite expresar el BANA en función de los efectos significativos (simples e interacciones dobles) que más contribuyen al modelo.

$$
\mathrm{BANA}=42.60-6.97 \mathrm{D}+10.85 \mathrm{AD}-3.40 \mathrm{BD}
$$

Se utiliza la ecuación (2) para optimizar y proyectar los valores de maximización dando como resultado un valor de estimación del BANA de 62.488.520 USD, cuya inversión inicial se recupera a una tasa superior a la TIO (índice de rendimiento, IR esperado $=1,12$ ), a partir del noveno año de puesta en marcha del proyecto. Los resultados también sugieren que la política de bodegaje dedicada con capacidad mínima de almacenamiento con una proyección del tamaño del CDC mínimo y con un sistema de facturación del servicio en función de la posición es la más apropiada para ser implementada. Sin embargo, de acuerdo a los resultados de significancia estadística, el diseñador puede elegir el tamaño proyectado del CDC, el criterio de costeo del servicio y la política de almacenamiento a conveniencia considerando la interacción significativa entre factores. 


\section{CONCLUSIONES}

De acuerdo a los resultados de este estudio llevado a cabo con datos de una empresa de griferías en Bogotá, se concluyó que el único factor estadísticamente significativo (con una significancia del 5\%) en el diseño de proyectos inmobiliarios de tipo industrial conducente a naves de almacenamiento inmóticas es la capacidad de bodegaje, que a su vez tiene interacciones dobles significativas con el tamaño proyectado del centro de distribución centralizado (CDC) y el criterio de costeo del servicio y que entre estos dos últimos también existe interacción significativa.

Siendo así, el diseño metodológico propuesto, con la inclusión de DOE, permite racionalizar la inversión en capital de trabajo al integrar, desde el inicio del diseño del edificio industrial, su operatividad (plan de operatividad), de acuerdo con reglas de decisión basadas en procesos de optimización. Para el caso específico estudiado se pudo proyectar un edificio que cumple los requerimientos, con el que se logra un beneficio actualizado neto ajustado (BANA) de 62.488.520 USD y se recupera la inversión aproximadamente al noveno año de puesta en marcha del proyecto.

\section{REFERENCIAS}

Abdul-Jalba, B.Y., M. Gutiérrez y J. Sicilia, Single cycle policies for the one- warehouse N-retailer, The International Journal of Management Science, 34, 196-208 (2006)

Albrecht, M., Supply chain coordination mechanisms: New approaches for collaborative planning, $1^{\text {a }}$ Ed., Springer Science \& Business Media, Berlin-Heidelberg, Germany (2010)

Altarazi, S.A. y M. Ammouri, Concurrent manual-order-picking warehouse design: a simulation-based design of experiments approach, International Journal of Production Research, 1-19 (2017)

Baker, P. y M. Canessa, Warehouse design: a structured approach, European Journal of Operational Research, 193 (2), 425-436 (2009)

Bartholdi III, J.J. y S.T. Hackman, Warehouse \& Distribution Science: Release 0.98, 5-36, Supply Chain and Logistics Institute, Atlanta, USA (2017)

Chackelson, C., A. Errasti, D. Ciprés y F. Lahoz, Evaluating Order Picking Performance Trade-offs by Configuring Main Operating Strategies in a Retail Distributor: A Design of Experiments Approach, International Journal of Production Research, 51 (20), 6097-6109 (2013)

Charvat, J.J., Project management methodologies: Selecting, Implementing, and Supporting Methodologies and Processes for Projects, $1^{\text {a }}$ Ed., 12-30, Jhon Wiley \& Sons, New York, USA (2003)

Czitrom, V., P. D. Spagon, Statistical Case Studies for Industrial Process Improvement ASA-SIAM on Statistics and Applied Probability, $1^{\text {a }}$ Ed., 171-298, SIAM-ASA, Philadelphia, USA (1987)

Diego-Más, A., Optimización de la distribución en planta de instalaciones industriales mediante algoritmos genéticos: Aportación al control de la geometría de las actividades, Tesis Doctoral, Valencia, España, UPV (2006)

Ekren B., S. Heragu, A. Krishnamurthy y C. J. Malmborg, Simulation based experimental design to identify factors affecting performance of AVS/RS, Computers \& Industrial Engineering, 58 (1), 175-185 (2010)

Gatignon, H., Statistical Analysis of Management Data, 3a Ed., 105-136, Springer, New York, USA (2014)

Gnimpieba, Z., A. Nait-Sidi-Moh., D. Durand y J. Fortin, Using Internet of Things technologies for a collaborative supply chain: Application to tracking of pallets and containers, Procedia Computer Science, 56, 550-557 (2015)

Hualpa Zúñiga, A. y C. Suarez Roldán, Ubicación y dimensionamiento como parámetros en el diseño de almacenes: revisión del estado de arte, Ingeniería., 18 (1), 65-83 (2013)

Jiménez, S., Estado del arte de los modelos matemáticos para la coordinación de inventarios en la cadena de suministro, Publicación Técnica, № 281, 9-31, Ciudad de México D.F., México, IMT de México (2005)

Kolaczyk, D., Statistical Analysis of Network Data: Methods and Models, $1^{\text {a }}$ Ed., 15-48, Verlag-Springer, New York, USA (2009)

Kuehl, R., Design of Experiments: Statistical Principles of Research Design and Analysis, $2^{\mathrm{a}}$ Ed., 320-375, Duxbury Press, Michigan, USA (1999)

Lahmar, M., Facility logistics: Approaches and solutions to next generation challenges, $1^{\text {a }}$ Ed., 17-60, Auerbach Publications, Kansas, USA (2008)

Mauleón, M., Sistemas de Almacenamiento y Picking, 1aㅡ Ed., 53-65, Díaz de Santos, Madrid, España (2013)

Mejía, G. y D. M., Franco-Duran, Evidence-based Improvement Policies in Public Management: A Case Study of Public Projects in Barrancabermeja City, Colombia, Journal of Construction in Developing Countries, 22(2), 55-66 (2017)

Mendes, Jr., Demand driven supply chain: A structured and practical roadmap to increase profitability, $1^{\text {a }}$ Ed., 5-24, Springer, Berlin, Germany (2011) 
Montgomery, D., Design and analysis of experiments, $8^{a}$ Ed., 304-318, Jhon Wiley \& Sons, Nueva Jersey, USA (2013)

Muckstadt, J. y R. Roundy, Multi-item, One-Warehouse, Multi-Retailer Distribution System, Management Science, 33, 1613-21 (1987)

Muther, R., Systematic Layout Planning, $1^{\text {a }}$ Ed., 15-25, Richard Muther \& Associates, Kansas, USA (1968)

Palacio-León, O. y W. Adarme-Jaimes, Coordinación de Inventarios: Un caso de estudio para la logística de ciudad, DYNA, 81(186), 295-303 (2014)

Palacio-León, O., Propuesta metodológica para el diseño y operación de instalaciones de almacenamiento modulares ecoeficientes para productos no perecederos, Tesis de Maestría, Bogotá D.C., Colombia, Universidad Nacional de Colombia (2012)

Palacio-León, O., Propuesta metodológica para la evaluación financiera de proyectos inmobiliarios de tipo industrial, Tesis Doctoral, Campeche, México, Universidad Internacional Iberoamericana, en impresión (2019)

Rakesh, V. y G. Adil, Layout optimization of a three dimensional order picking warehouse, IFAC-PapersOnLine, 48(3), 1155-1160 (2015)

Restrepo, J. y G. Uribe, Análisis económico de plantas industriales, DYNA, 134, 53-63 (2002)

SPSS Statistics 21, Global system for data analysis. New York: IBM Corporation (2013)

Sule, D.R., Manufacturing Facilities: Location, Planning, and Design, $3^{a}$ Ed., 441-445, CRC Press, Nueva Jersey, USA (2008)

Tersine, R. J., Principles of inventory and materials management, $1^{\text {a }}$ Ed., 3-27, Prentice-Hall, New York, USA (1994)

Tompkins, J. A., J. A. White e Y.A. Bozer, Facilities planning, 4ª Ed., 113-126, John Wiley \& Sons, New York, USA (2010)

Vélez-Pareja, I. A., Decisiones de inversión: para la valoración financiera de proyectos y empresas, 5토 Ed., 182-186, Editorial Pontificia Universidad Javeriana, Bogotá, Colombia (2013) 